\title{
Effectiveness of Jacobson's Progressive Muscle Relaxation (JPMR) on Hypertension among School going Adolescents
}

\author{
Rajagopal Manjushambika ${ }^{1}$, Baby Prasanna $^{2}$, R. Vijayaraghavan ${ }^{3}$, Bai Sushama $^{4}$ \\ ${ }^{1}$ Ph.D. in Nursing, Department of Research Saveetha University, Chennai, ${ }^{2}$ Ph.D. in Nursing, Professor and \\ Former Principal of Sri Ramachandra College of Nursing Porur, ${ }^{3}$ Ph.D. Director of Research, Saveetha \\ University, ${ }^{4}$ Former Head of the Department, Pediatrics; Pushpagiri Medical College, Thiruvalla
}

\begin{abstract}
Background: Hypertension could have its origin in childhood and go undetected unless specially looked for. Though many risk factors were postulated for hypertension among adolescents, gap in knowledge is that no studies are reported on effectiveness of interventions for hypertension among adolescents.

Objectives: To determine: 1) effectiveness of JPMR on blood pressure. 2) association of blood pressure to selected variables. Basic procedure: In first phase, a cross-sectional survey was conducted among 980 adolescents following multistage stratified random sampling from classes $6^{\text {th }}$ to $12^{\text {th }}$. Anthropometric measurements, HR, BP and selected blood tests were done. In second phase experimental approach with pre-test, post-test control and experimental was done with JPMR as intervention.
\end{abstract}

Main Findings: Both rural and urban adolescents were in par with regard to prevalence of hypertension. There was significant association between hypertension and family history. Prevalence of hypertension among 14-17 years was higher than 11-13 years. Prevalence of hypertension among overweight and obese adolescents was significantly higher than their counterparts. Mean post-test systolic and Diastolic BP of experimental groups in both rural and urban areas were significantly lower than their pre-test values and the post-test values of their counterparts suggesting JPMR was effective.

Conclusion: JPMR is an effective intervention for adolescent hypertension. Relaxation techniques can be made as part of curriculum in schools.

Keywords: Jacobson's Progressive Muscle Relaxation, Heart Rate,Hypertension, Body Mass Index, Fasting Lipid Profile, School going Adolescents.

\section{Introduction}

Increased blood pressure (BP) in childhood has been considered a risk factor for hypertension in adulthood. According to non-communicable diseases (NCD) country profile in India, the proportional mortality from NCD is $60 \%$; of which cardiovascular diseases account for $26 \%$. The sixth target in the Global NCD action plan calls for $25 \%$ reduction in this. ${ }^{(1)}$

\footnotetext{
Corresponding Author:

Dr. Rajagopal Manjushambika

Ph.D. in Nursing, Department of Research Saveetha University, Chennai

e-mail: mksr200503@yahoo.com
}

Background of the study: Adolescent hypertension is a growing health problem. Early diagnosis is an important strategy in its control and prevention of complications. Adolescents with BP levels in higher portion of BP distribution curve tend to maintain that position over time, which is indicative of BP tracking (2). Among adolescents, $85 \%-95 \%$ of hypertension is primary hypertension which could easily be managed by lifestyle modifications.

During a child's hospital visit, blood pressure is not measured. Also at schools during the periodic health checkup only anthropometry is measured. Unlike in adults, normal blood pressure in children vary according to their age, gender and height ${ }^{(3)}$. WHO (2007) fact sheet recommends interventions 
for early detection, prevention and management of hypertension $^{(4)}$.A study of 1022 students aged 14-19 years in New Delhi, showed prevalence of hypertension was $6.4 \%^{(5)}$.Study in Kerala among 20263 students, incident hypertension was $11 \%$.Systolic hypertension was $5.84 \%$ and diastolic hypertension was $6.61 \%$. $10.65 \%$ had systolic pre-hypertension and $14.75 \%$ had diastolic pre-hypertension ${ }^{(6)}$. Cross sectional survey among 400 adolescents in Chennai revealed $21.5 \%$ was hypertensive ${ }^{(7)}$. Study conducted among 410 adolescent in Nagpur revealed $15.9 \%$ were pre hypertensive and $13.9 \%$ were hypertensive ${ }^{(8)}$. Study among 400 adolescents revealed $24.4 \%$ prevalence of high blood pressure ${ }^{(9)}$. Globalization is bringing more lifestyle modifications. Adolescents are exposed to multiple risk factors including obesity, unhealthy eating habits, academic stress etc. No studies are reported on effectiveness of relaxation for hypertension among adolescents.

Objectives: Aim of study was to determine effectiveness of JPMR on hypertension among adolescents. The projected outcome of study was control of hypertension among adolescents and thereby preventing the chance of stroke, cardiovascular and renal diseases in future.Objectives of the study were to determine:

(1) Effectiveness of JPMR onheart rate (HR), systolic blood pressure (SBP) and diastolic blood pressure (DBP).

(2) Association of blood pressure to selected variables.

\section{Materials and Method}

Inclusion Criteria: 11-17 years having pre, stage-I and stage-II hypertension.

Exclusion Criteria: Any medical condition not allowing practicing JPMR.

Sample and sample size: Alappuzha district has four educational districts, of which one was selected. After pilot study,prevalence of hypertension was $29 \%$, based on which sample size for first phase was calculated to be 940 , for a relative precision of $90 \%$ at $5 \%$ significance level.

Sampling Technique: Multistage stratified random sampling technique was used. There were 20 mixed rural Government Higher Secondary Schools (HSS), and 7 mixed urban Government HSS. Considering 25\%,
5 rural 2 urban schools were selected. 140 students from each school, taking 20 students each from standard $6^{\text {th }}$ to $12^{\text {th. }}$; made the sample size to be 980 . Participants were selected from the selected divisions. For the second phase, considering a reduction of $8 \mathrm{mmHg}$ in systolic pressure and $10 \mathrm{~mm} \mathrm{Hg}$ as combined standard deviation, 90\% power and 5\% significance, estimated sample size was 34 ; considering $10 \%$ as attrition the sample size was rounded off to 40 per group. (Sample size calculated by Sigma Plot 13, Systat Software, USA).

Participants: For the first phase, 980 adolescents. For the second phase, all identified participants from one rural and urban school each were assigned as control and experimental groups.

\section{Data collection tools:}

Anthropometry: Height, weight, body mass index (BMI), waist circumference (WC), hip circumference (HC) and waist hip ratio (WHR) were measured .Height was measured to nearest centimeter using tape measure and noted in meters. Weight was measured using calibrated balance scale and noted in kilograms.BMI was calculated as ratio of weight in kilogram by the square of height in meters. BMI interpretation by WHO was used for classification ${ }^{(10)}$. WC was measured using flexible tape over the abdomen, with measurements made halfway between lower border of ribs and highest point of iliac crest, while standing, at end of normal expiration. $\mathrm{HC}$ was measured at widest point over the buttocks when viewed from side. WHR was calculated by dividing $\mathrm{WC}$ by $\mathrm{HC}$.

Blood pressure and heart rate: HR was counted for one full minute. BP was measured using calibrated mercury sphygmomanometer. Measurements were taken on right arm supported at heart level after sitting for five minutes. Average of all three readings was taken as final observation. The participants were classified according to NHBPEP ${ }^{(3)}$.

Background data, physical activity, eating habits questionnaire: Physical activities were assigned metabolic equivalent of task (MET) values based on compendium of physical activity ${ }^{(11)}$ and compendium of physical activity for youth ${ }^{(12)}$. Data on eating habits of one week was collected using a 5 point likert scale ${ }^{(13)}$. The reliability of physical activity questionnaire was 0.80 and that of eating habits questionnaire was 0.81 .

Biochemical profile: Serum creatinine was 
measured for all identified hypertensive cases ${ }^{(14)}$. Lipid profile and TSH were measured for subset of sample.

Jacobson's progressive muscle relaxation technique (JPMR): JPMR includes progressive contraction and release of entire muscle groups of body. First individual muscle group will be contracted and feel tension followed by releasing same muscles using an audio commentary, under supervision. Participants sat comfortably on chairs, closing eyes, bringing attention to their breathing ${ }^{(15)}$.

Data collection procedure: The study was conducted after obtaining approval from Ethics Committee of Saveetha University, permission from District Director of Education (DDE) and Head Mistress of schools. Assent and informed consent were obtained from participants and their parents respectively.

Phase-I Descriptive survey method: Anthropometric measurements, HR, BP, background data, data on physical activity and eating habits were collected.

Phase-II Experimental method: Experimental approach with before and after with control design was used. Experimental group was taught and practiced JPMR for 20 min daily for 3 weeks. Post-test measurements were taken.

\section{Results}

Prevalence of hypertension among adolescents: Table 1 shows prevalence in rural and urban area. The obtained $\chi^{2}$ value was not significant. Both rural and urban adolescents are in par with regard to prevalence. Distribution of age and hypertension among adolescents in rural and urban areas is shown in fig: 1 . Prevalence among 14-17 years in rural and urban areas (about 35\% and 36\%) was higher than 11-13 years in rural and urban areas (about 22\% and 20\%) and was statistically significant $(\mathrm{p}<0.001)$.Similarly, prevalence among adolescents in class IX-XII in rural and urban areas were similar; (about 34\% each) and was higher than that of adolescents in class VI-VIII in rural and urban areas (about $22 \%$ and $21 \%$ respectively) and was statistically significant $(\mathrm{p}<0.001)$. There was significant association between hypertension and family history (0.009) and there was no significant association between hypertension and gender, WHR, food habits, physical activity and thyroid disease.
Distribution of BMI and hypertension among adolescents in rural and urban areas is shown in fig: 2 Prevalence of hypertension among adolescents in BMI category of II (Z scores more than I; risk of overweight and obesity) in rural and urban areas (about $54 \%$ and $53 \%$ respectively) was higher than prevalence of hypertension among adolescents in the BMI category of I ( $Z$ scores less than I; normal,wasted) in rural and urban areas (about $26 \%$ and $23 \%$ respectively) and was statistically significant $(\mathrm{p}<0.001)$.

Effectiveness of JPMR on HR: Mean and standard error of HR, SBP and DBP of rural and urban adolescents are given in table 2 . The mean difference of post-test HR of rural control and experimental groups was statistically significant $(p=0.005)$. In urban areas also, mean difference of post-test HR of control and experimental groups was statistically significant $(\mathrm{p}=0.045)$; showing JPMR was effective in reducing HR among both rural and urban adolescents.

Effectiveness of JPMR on blood pressure: Mean post-test SBP of rural experimental group (118.7) was $10 \mathrm{mmHg}$ lower than their mean pre-test SBP (128.4) and was statistically significant $(p<0.001)$. The mean difference of post-test SBP of control and experimental groups was also statistically significant $(\mathrm{p}<0.001)$. Mean post-test SBP of urban experimental group (115.0) was $8 \mathrm{mmHg}$ lower than their mean pre-test SBP (123.4) and was statistically significant $(\mathrm{p}<0.001)$. Mean difference of post-test SBP of control and experimental groups was also statistically significant $(\mathrm{p}<0.001)$. The results showed that JPMR was effective in reducing SBP among both rural and urban adolescents.

Figure 3 shows effectiveness of JPMR on DBP in rural areas. Mean post-test DBP of experimental group (73.0) was $5 \mathrm{mmHg}$ lower than their mean pre-test DBP (78.0) and was statistically significant $(\mathrm{p}<0.001)$. Mean difference of post-test DBP of control and experimental groups was also statistically significant $(p<0.001)$; showing JPMR was effective in reducing DBP among rural adolescents. Figure 4 shows effectiveness of JPMR on DBP in urban areas. Mean post-test DBP of experimental group (71.4) was $8 \mathrm{mmHg}$ lower than their mean pre-test DBP (79.1) and it was statistically significant $(p<0.001)$. The mean difference of posttest DBP of control and experimental groups was also statistically significant $(p<0.001)$; showing JPMR was effective in reducing DBP among urban adolescents. 
Distribution of fasting lipid profile: One third (37\%) of adolescents had increased serum total cholesterol values. Obtained chi-square value was not statistically significant $(\mathrm{p}=0.774)$. Majority of adolescents in rural (86\%) and in urban (88\%) had low serum HDL values. Obtained chi-square value was not statistically significant $(\mathrm{p}=0.790)$. High LDL levels were seen more among urban adolescents (56\%) compared to rural adolescents (40\%). Obtained chi-square value was not statistically significant $(\mathrm{p}=0.436)$. In summary, there was no significant difference between rural and urban adolescents with regard to fasting lipid profile values.

Table 1: Prevalence of hypertension among rural and urban adolescents

\begin{tabular}{|l|c|c|c|}
\hline Type of Hypertension & Rural $(\mathbf{N}=\mathbf{7 0 0})$ & Urban $(\mathbf{N}=\mathbf{2 8 0})$ & $\mathbf{X}^{\mathbf{2}}$ (p value) \\
\cline { 1 - 3 } PHT & $95(47.26)$ & $45(56.25)$ & $3.010(\mathrm{p}=0.222)$ \\
\cline { 1 - 3 } Stage-1 HTN & $97(48.26)$ & $34(42.5)$ & $1(1.25)$ \\
\hline Stage-2 HTN & $9(4.48)$ & \\
\hline
\end{tabular}

Table 2: Mean \pm SE of heart rate, systolic and diastolic blood pressure of rural and urban adolescents

\begin{tabular}{|c|c|c|c|c|}
\hline \multirow{2}{*}{ SI } & \multirow{2}{*}{ Parameter } & \multicolumn{2}{|c|}{ Rural } & \multirow{2}{*}{$\begin{array}{c}\text { Urban } \\
\text { Mean } \pm \text { SE }\end{array}$} \\
\hline & & Groups & Mean \pm SE & \\
\hline \multirow{4}{*}{1} & \multirow{4}{*}{ HR } & Control Pre-test & $82.2 \pm 0.9$ & $83.5 \pm 1.5$ \\
\hline & & Control Post-test & $80.4 \pm 0.8$ & $80.3 \pm 1.5$ \\
\hline & & Exp. Pre-test & $81.7 \pm 1.0$ & $82.6 \pm 1.4$ \\
\hline & & Exp. Post-test & $77.1 \pm 0.7$ & $76.8 \pm 0.9$ \\
\hline \multirow{4}{*}{2} & \multirow{4}{*}{ SBP } & Control Pre-test & $125.3 \pm 1.1$ & $122.4 \pm 1.1$ \\
\hline & & Control Post-test & $124.6 \pm 0.8$ & $123.3 \pm 1.2$ \\
\hline & & Exp. Pre-test & $128.4 \pm 1.3$ & $123.5 \pm 1.2$ \\
\hline & & Exp. Post-test & $118.7 \pm 0.7$ & $115.0 \pm 1.1$ \\
\hline \multirow{4}{*}{3} & \multirow{4}{*}{ DBP } & Control Pre-test & $76.9 \pm 0.8$ & $77.3 \pm 1$ \\
\hline & & Control Post-test & $76.9 \pm 0.6$ & $77.9 \pm 1$ \\
\hline & & Exp. Pre-test & $78.0 \pm 1.1$ & $79.1 \pm 0.8$ \\
\hline & & Exp. Post-test & $73.0 \pm 0.7$ & $71.4 \pm 0.8$ \\
\hline
\end{tabular}

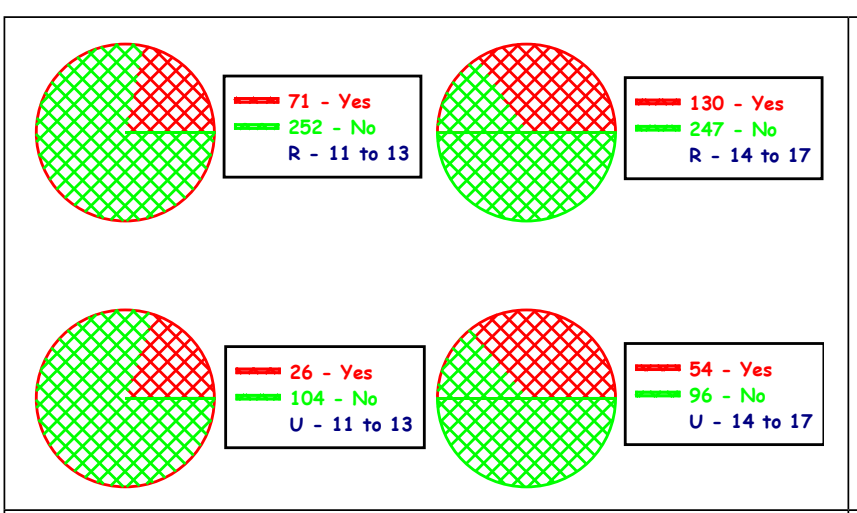

Figure 1: Age distribution of hypertensive adolescents in rural $(R)$ and urban $(U)$ areas.

$\mathrm{N}-$ Rural $=700 ;$ Urban $=280$.

Rural $-\chi 2=12.678, \mathrm{P}<0.001$. Urban $-\chi 2=7.970, \mathrm{P}<0.001$.

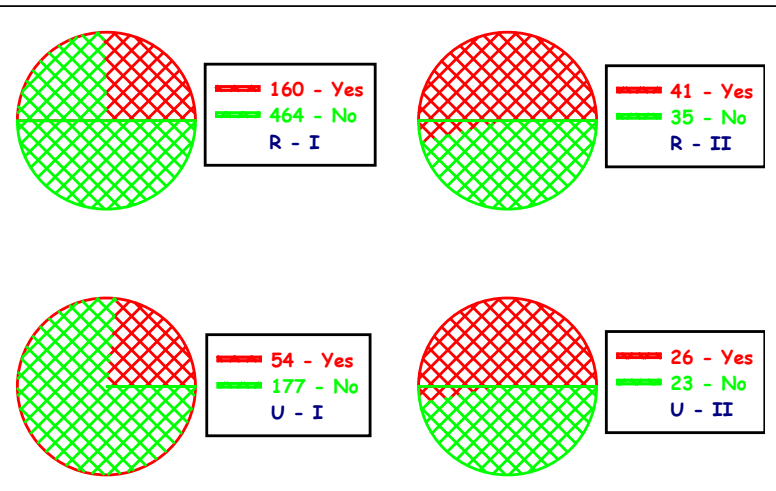

Figure 2: Distribution of BMI (I-Z scores below1; II-Z scores above1) of hypertensive adolescents

$\mathrm{N}-$ Rural $=700 ;$ Urban $=280$.

Rural $-\chi 2=25.155, \mathrm{P}<0.001$. Urban $-\chi 2=16.030, \mathrm{P}<0.001$. 


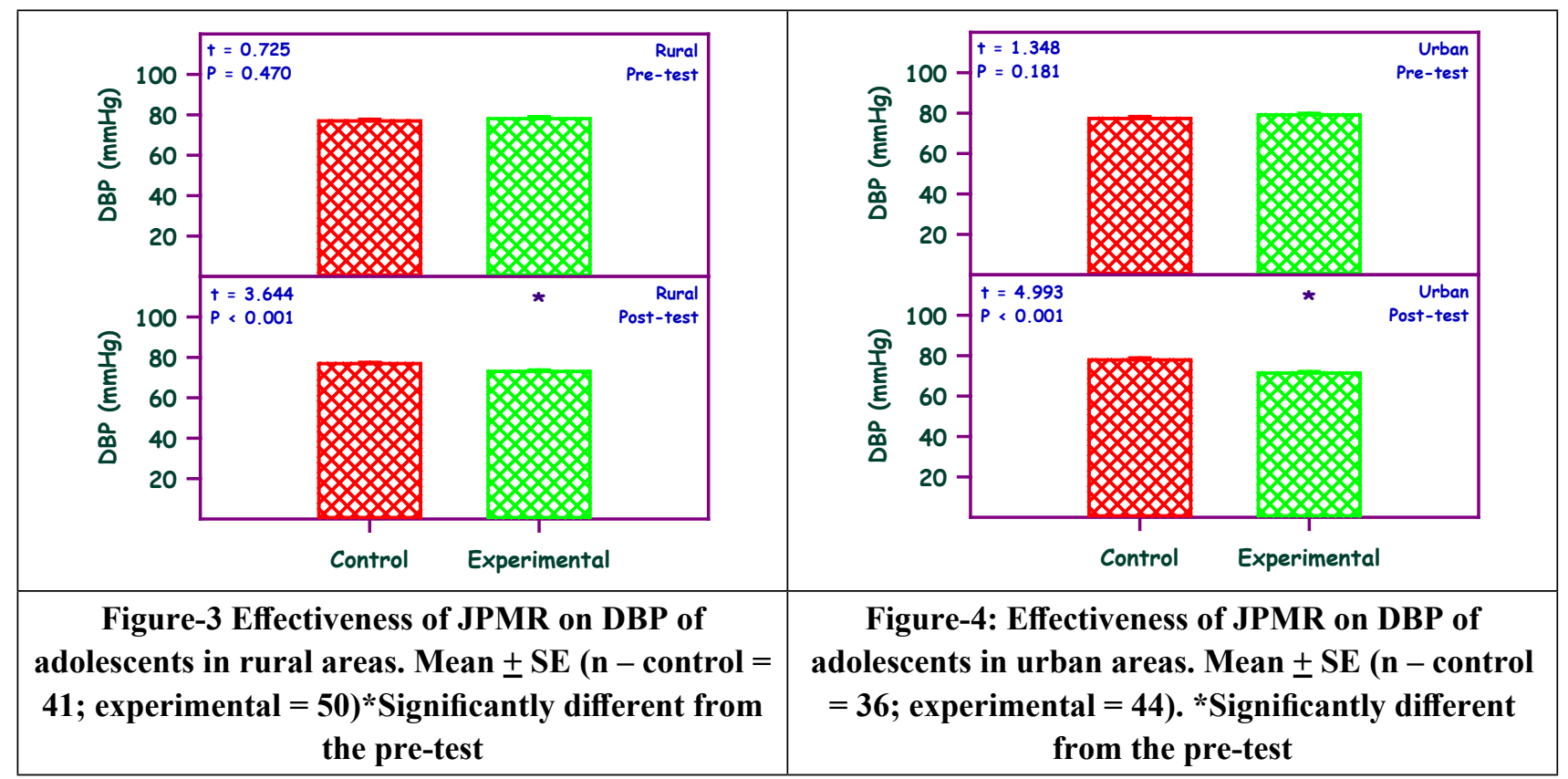

\section{Discussion}

Present study findings showed that prevalence of hypertension among rural and urban adolescents was $28.7 \%$, and $28.57 \%$ respectively. Prevalence of adolescent hypertension in early 2000 was about $3-6 \%$; by 2010 studies reported about $12-16 \%$ of hypertension, it had increased further to about $25 \%$ by $2016^{(18,6,19,16,17)}$. Another study among 400 adolescents reported 24.4\% prevalence of high blood pressure, which is slightly lower than present study findings $(28.97 \%){ }^{(20)}$.Present study results showed significant association between hypertension and family history, age, class of study and BMI. A study conducted in Gaziabad among 1340 adolescents showed significant association between hypertension and BMI, salt intake and positive family history $^{(21)}$. A study done among 1005 adolescents reported significant association with hypertension and positive family history and BMI ${ }^{(20)}$. The findings showed that adolescent hypertension is a growing health problem that needs immediate measures to reduce or prevent the further increase.

\section{Conclusion}

The study findings showed JPMR was effective in reducing BP among adolescents. Though many studies reported prevalence of hypertension among adolescents, no interventions are yet reported for same. Present study finding strongly recommends need for preventive measures focused on adolescents to avoid hypertensive epidemic in them.
Acknowledgement: Authors are thankful to study participants, DDE and Head of schools for granting permission

Conflicting Interest: Authors declare no competing interests.

Contribution Details: All authors have contributed substantially towards publishing article.

\section{References}

1. World Health Organisation. Global Action Plan for Prevention and Control of Non Communicable Diseases 2013-2020. (https://www.who.int/ publications/i/item/9789241506236).

2. Flynn JT, Falkner BE. Obesity hypertension in adolescents: Epidemiology, Evaluation, and Management. The Journal of Clinical Hypertension. 2011; 13(5):323-331.

3. National High Blood Pressure Education Program Working Group on High Blood Pressure in Children and Adolescents. The fourth report on the diagnosis, evaluation, and treatment of high blood pressure in children and adolescents. Pediatrics. 2005; 114 (2): 555-576.

4. WHO Fact sheet on cardiovascular diseases 2007.

5. Agarwal SK, Goel R, Misra A and Vikram N. Correlates of hypertension among urban Asian Indian adolescents. Archives of disease in child hood. 2010;95 (12): 992-997. 
6. Raj M, Sundaram KR, Paul M, Deepa AS and Kumar RK. Obesity in Indian children: Time trends and relationship with hypertension. Natl Med J India. 2007; 20(6): 288-293.

7. Sundar JS, Adaikalam JMS, Parameswari S, Valarmarthi S, Kalpana Sand Shantharam D. Prevalence and Determinants of Hypertension among Urban School Children in the Age Group of 13- 17 Years in Chennai, Tamilnadu. Epidemiology. 2013; 3:130.

8. Desh Pande AV. Study of prevalence of hypertension in adolescent in Central India. International Journal of Basic Medicine and Clinical Research. 2014; 1(3): 66-70.

9. Bhalavi V, Deshmukh P, Akram M and Mahajan B. Study of hypertension and hyperlipidemia in the adolescent of Central India. International Journal of Recent Trends in Science and Technology. 2014; 10 (3): 495-498.

10. World health Organisation .Growth reference data for 5- 19 years. 2007.

11. Ainsworth BE, Haskell WL, Herrmann SD, Meckes N, Bassett DR Jr, Tudor-Locke Cetal.. Compendium of physical activities: a second update of codes and MET values. Medicine and Science in Sports and Exercise. 2011; 43(8): 1575-1581.

12. Ridley K, Ainsworth BE and Olds ST. Development of a compendium of energy expenditure for youth. The International Journal of behavioral nutrition and Physical Activity. 2008; 5: 45.

13. Hazza MH, Hana IA and Abdulrahman OM . Convergent validity of the Arab Teens Life style Study (ATLS) Physical Activity Questionnaire. International Journal of Environmental Research and Public Health. 2011; 8: 3810-3820.
14. Luma GB and Spiotta RT. Hypertension in children and adolescents. American Family Physician. 2006; 73(9):1558-1666.

15. Institute for Psychotherapy and Management Science. Jacobson's Progressive Muscle Relaxation. Text book on psychotherapy Mumbai. 2004; 92-97.

16. Tony L, Areekal B, Nair ATS, Ramachandran R, Philip RR, Rajasi RS, et al. Prevalence of hypertension and pre-hypertension among adolescent school children in Thiruvananthapuram, Kerala, India. Int J Community Med Public Health. 2016; 3(12):3556-3563.

17. Naha NK, John M, Cherian VJ. Prevalence of hypertension and risk factors among school children in Kerala, India. Int J Contemp Pediatr. 2016; 3.

18. Soudarssanane M.B, Karthigeyan M, Stephen $\mathrm{S}$ and Sahai A. Key Predictors of High Blood Pressure and Hypertension among Adolescents: A Simple Prescription for Prevention. Indian Journal of Community Medicine. 2006; 31(3).

19. MadhavikuttyAmma GD, Vasudevan B, Akshayakumar S. Prevalence and determinants of prehypertension and hypertension among adolescents: a school based study in a rural area of Kerala, India. Int J Res Med Sci. 2015;3: 58-64.

20. Kavitha B, Bhuvaneswari $\mathrm{K}$ and Vinoth $\mathrm{P}$. Prevalence of sustained hypertension among adolescent school children in Puducherry. Indian Journal of Basic and Applied Medical Research. 2016; 5(4) 649-655.

21. Gupta GK, Agarwal D, Singh RK and Arya R.K. Prevalence, risk factors and socio demographic co-relates of adolescent hypertension in district Ghaziabad. Indian journal of community health. 2013; 25(3): 293-298. 